\title{
Refraction-Assisted Illumination for Infrared Imaging of Integrated Circuits
}

\author{
Stephen LaLumondiere, Terrence Yeoh* \\ Photonics Technology Department, Physical Sciences Laboratory, The Aerospace Corporation \\ *Microelectronics Technology Department, Physical Sciences Laboratory, The Aerospace Corporation
}

We describe a technique in which a broadband IR illumination source is placed such that the light is incident at approximately $45^{\circ}$ with respect to the surface normal at a point directly beneath a collection objective of a microscope system. This ensures that, for a reasonably smooth surface, the bidirectional reflectance distribution function (BRDF) will have a strong component at $45^{\circ}$ with respect to the surface normal, and the majority of the surface component will be reflected or scattered out of the field of view of the imaging objective. For a material with a large index of refraction, as is the case with silicon ( $\mathrm{n}=3.5$ @ $1.1 \mu \mathrm{m}$ ), the transmitted light is refracted inside the silicon at an angle of approximately $12^{\circ}$ with respect to the surface normal. This refracted light can eventually be scattered back towards the top surface if it strikes a highly effective scattering site such as a metal line. Any light that is returned slightly off normal will be either refracted away at an angle which does not fall within the acceptance cone of the objective, or experience total internal reflection within the structure, as shown in Fig 1. The combination of spatial filtering of the surface scattered light and the reciprocity of Snell's law as applied to high index of refraction materials results in a high-contrast, high-resolution image using this refraction-assisted illumination technique.

This filtering is due to an effect well known by scuba divers in which the field of view of the surrounding environment is compressed into a small window known as "Snell's window." In this case, Snell's window provides two main advantages for high-resolution imaging. For high index of refraction materials such as silicon, Snell's window is quite small $\left(16^{\circ}\right)$, and any reflected light from the second surface outside of this window will experience total internal reflection. Through multiple scattering events this light will provide the diffuse, omnidirectional light necessary to evenly illuminate the underlying features. However, for the light that is scattered within Snell's window, the effect provides additional resolution gains. This is due to the fact that for imaging, Snell's window acts as an additional spatial filter that filters the reflected light angularly and only permits the normal components to exit and be collected by the objective lens. As a result, all the light collected by the lens will be nearly parallel to the surface normal. Light scattered at angles off from the surface normal from separate areas of the region of interest that would normally be coincident on other parallel areas and will be refracted away from the objective lens. 

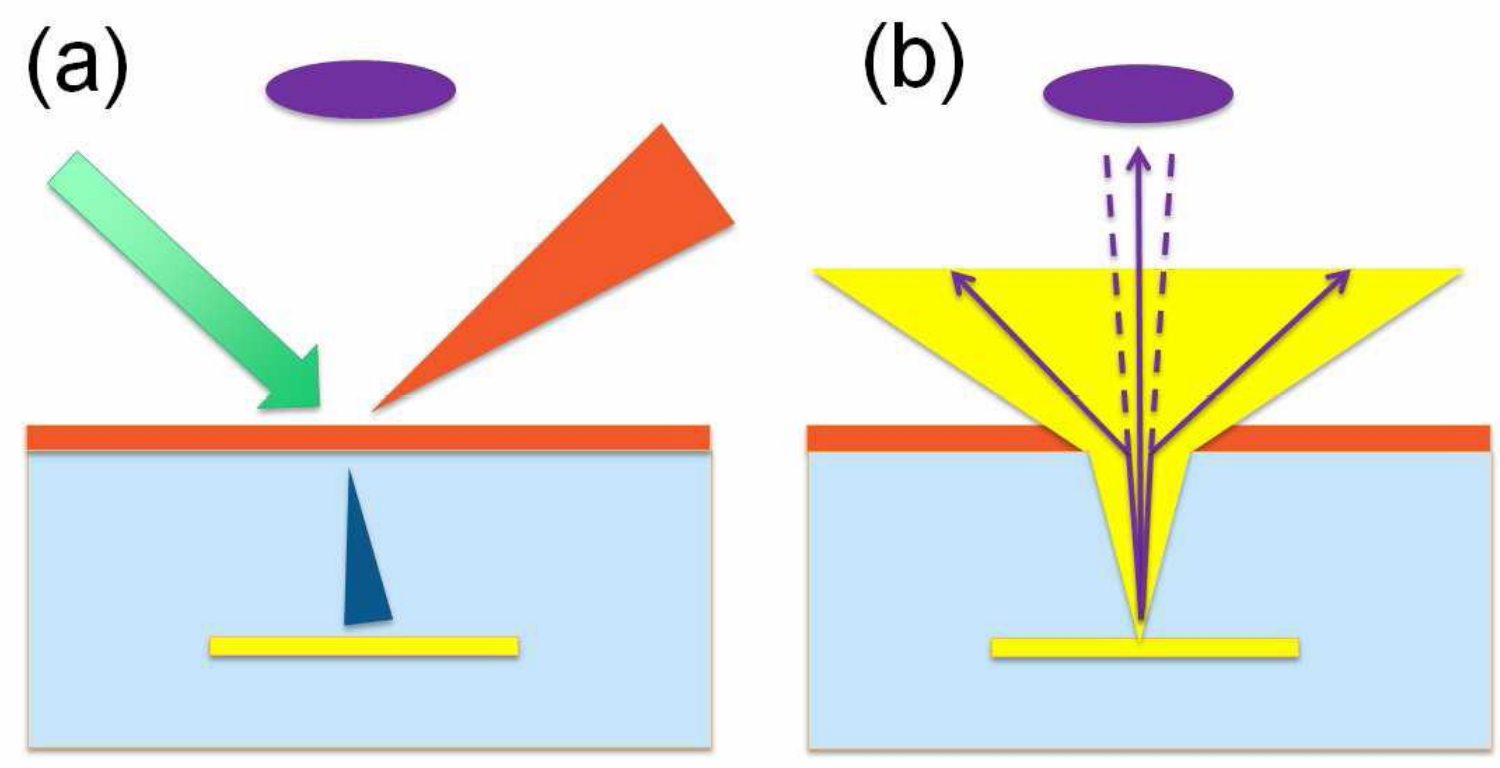

Fig. 1. (a) Incident light (green) is reflected in a range of angles modeled as a cone (orange) as a result of surface roughness. The reflected light is not collected by the objective lens (purple). Some component will also be transmitted and refracted over a range of angles (blue), and will strike the underlying metallization (yellow). (b) Light reflected from the metallization (yellow) will return over a range of angles dependent on the second surface roughness. Only the components near normal to the top surface will be collected by the objective lens.

The simplicity of the microscope will allow it to be compactly integrated into other instruments and tools that require backside IR imaging in confined spaces. Systems such as focused ion beam systems and laser assisted chemical etch will benefit from this improved resolution. This technique can also be expanded to other high-index materials such as GaAs and InP, and represents a new class of side-illumination microscopes that takes advantage of Snell's window to achieve high-resolution, high-contrast images.

This work was supported under The Aerospace Corporation's Mission Oriented Investigation and Experimentation program, funded by the U.S. Air Force Space and Missile Systems Center under Contract No. FA8802-09-C-0001. 